\title{
A Randomized Clinical Trial of Acceptance and Commitment Therapy Versus Progressive Relaxation Training for Obsessive-Compulsive Disorder
}

\author{
Michael P. Twohig \\ Utah State University
}

\author{
Steven C. Hayes, Jennifer C. Plumb, Larry D. Pruitt, \\ Angela B. Collins, and Holly Hazlett-Stevens \\ University of Nevada, Reno
}

\author{
Michelle R. Woidneck \\ Utah State University
}

\begin{abstract}
Objective: Effective treatments for obsessive-compulsive disorder (OCD) exist, but additional treatment options are needed. The effectiveness of 8 sessions of acceptance and commitment therapy (ACT) for adult OCD was compared with progressive relaxation training (PRT). Method: Seventy-nine adults (61\% female) diagnosed with OCD (mean age $=37$ years; $89 \%$ Caucasian) participated in a randomized clinical trial of 8 sessions of ACT or PRT with no in-session exposure. The following assessments were completed at pretreatment, posttreatment, and 3-month follow-up by an assessor who was unaware of treatment conditions: Yale-Brown Obsessive Compulsive Scale (Y-BOCS), Beck Depression Inventory-II, Quality of Life Scale, Acceptance and Action Questionnaire, Thought Action Fusion Scale, and Thought Control Questionnaire. Treatment Evaluation Inventory was completed at posttreatment. Results: ACT produced greater changes at posttreatment and follow-up over PRT on OCD severity (Y-BOCS: ACT pretreatment $=24.22$, posttreatment $=12.76$, follow-up $=11.79 ;$ PRT pretreatment $=25.4$, posttreatment $=18.67$, follow-up $=16.23$ ) and produced greater change on depression among those reporting at least mild depression before treatment. Clinically significant change in OCD severity occurred more in the ACT condition than PRT (clinical response rates: ACT posttreatment $=46 \%-56 \%$, follow-up $=46 \%-66 \%$; PRT posttreatment $=13 \%-18 \%$, follow-up $=16 \%-18 \%)$. Quality of life improved in both conditions but was marginally in favor of ACT at posttreatment. Treatment refusal (2.4\% ACT, $7.8 \%$ PRT) and dropout $(9.8 \%$ ACT, $13.2 \%$ PRT) were low in both conditions. Conclusions: ACT is worth exploring as a treatment for OCD.
\end{abstract}

Keywords: acceptance and commitment therapy, obsessive-compulsive disorder, treatment, experiential avoidance, psychological flexibility

There is a need to develop new approaches to the treatment of obsessive-compulsive disorder (OCD). Exposure with ritual prevention (ERP) and ERP with cognitive procedures (i.e., cognitive therapy, or ERP-CT) are recognized as well-supported treatment methods that have a clinically significant impact on the majority of patients (Abramowitz, Taylor, \& McKay, 2009; Fisher \& Wells, 2005), but they raise various issues beyond clinical impact for

Michael P. Twohig and Michelle R. Woidneck, Department of Psychology, Utah State University; Steven C. Hayes, Jennifer C. Plumb, Larry D. Pruitt, Angela B. Collins, and Holly Hazlett-Stevens, Department of Psychology, University of Nevada, Reno.

The present project was funded by National Institute of Mental Health Grant R21 MH074968 to Steven C. Hayes. It was also conducted as Michael P. Twohig's dissertation at the University of Nevada, Reno, for which he received the Virginia Roswell Dissertation Award from the Association for Behavioral and Cognitive Therapies. We would like to thank Tami Jeffcoat, Heidi LaBash, Michael Levin, Lindsay Martin, Kate Morrison, Dahlia Mukherjee, and Kimberly Pratt for their assistance with this project. We would also like to thank Maureen Whittal for her comments on the article.

Correspondence concerning this article should be addressed to Michael P. Twohig, Department of Psychology, Utah State University, 2810 Old Main Hill, Logan, UT 84322-2810. E-mail: michael.twohig@usu.edu those accessing care. ERP suffers from a dropout rate of approximately 25\% (Abramowitz, Taylor, \& McKay, 2009), and overt refusal rates, though often not reported in the literature, represent an additional 5\%-22\% of participants (e.g., Foa et al., 2005; McLean et al., 2001). ERP and ERP-CT are intensive treatments. The ERP studies covered in a recent review (Abramowitz, Taylor, \& McKay, 2009) averaged $27.4 \mathrm{hr}$ of direct therapist contact in addition to dozens of hours of client homework. ERP is also grossly underutilized (Olatunji, Deacon, \& Abramowitz, 2009), perhaps in part because clinicians find it aversive (Richard \& Gloster, 2007) and the public rates it negatively when the methods are described (Richard \& Gloster, 2007). Finally, OCD tends to be a complex disorder with the majority of patients also having comorbid unipolar mood disorders, other anxiety disorders, or substance use disorders (Abramowitz, Taylor, \& McKay, 2009), suggesting that narrowly focused treatments might require augmentation to deal with all presenting clinical syndromes.

Merely the act of exposing oneself to a feared situation and staying in that situation for a meaningful period is generally therapeutic (Abramowitz, 1996), but there is no consensus about the psychological processes that account for this benefit (Steketee \& Barlow, 2002). Contemporary animal research suggests that extinction works by fostering new behaviors and new learning 
rather than unlearning (e.g., Bouton, 2002), which is consistent with the idea that exposure may work in part by fostering greater flexibility in responding in the presence of previously repertoirenarrowing stimuli such as anxiety, obsessive thoughts, or physical sensations. Arch and Craske (2008), for example, postulated that exposure involves "optimizing learning ... based on increasing tolerance for fear and anxiety" (p. 269). If that is a process of importance, however, there may be additional methods of fostering response flexibility without exposure in a formal, procedural sense. This in turn might help avoid the dropout and refusal problems that are commonly experienced with traditional exposure methods (cf. Levitt, Brown, Orsillo, \& Barlow, 2004), and may increase the overall efficiency of treatment.

From its earliest explication as a treatment for anxiety disorders, acceptance and commitment therapy (ACT; Hayes, Strosahl, \& Wilson, 1999) has been said to be a method that is exposure based in the sense that it "gives people an opportunity to practice experiencing anxiety without also struggling with anxiety" (Hayes, 1987, p. 365). In ACT theory, this approach comes under the general term psychological flexibility (Hayes, Luoma, Bond, Masuda, \& Lillis, 2006): engaging in positive behaviors rather than attempting to avoid difficult experiences and emotions. A lack of psychological flexibility is associated with OCD symptom levels in adults and children (Abramowitz, Lackey, \& Wheaton, 2009; Briggs \& Price, 2009). ACT seeks to establish psychological flexibility by targeting six major processes: acceptance (willingness to contact inner experiences), defusion (experiencing cognition as an ongoing process rather than allowing cognition to overly regulate behavior), self as context (experiencing oneself as the context in which inner experiences occur, rather than being defined by the content of experiences), being present (being able to flexibly attend to inner and outer events as they occur, nonjudgmentally), values (choosing desired consequences of ongoing patterns of behavior so as to establish reinforcers in the present), and committed action (flexibly moving in a valued direction). Component studies of these processes in isolation (outside the entire treatment package) support their utility in a variety of contexts (e.g., Levitt et al., 2004; Masuda, Hayes, Sackett, \& Twohig, 2004). A number of studies show that ACT impacts psychological flexibility and produces positive outcomes when psychological flexibility increases (e.g., Hayes et al., 2006).

It seems possible that ACT could be helpful in accomplishing these goals with OCD. There is evidence for the effectiveness of ACT in the treatment of OCD spectrum disorders such as skin picking and trichotillomania (Twohig, Hayes, \& Masuda, 2006b; Twohig \& Woods, 2004; Woods, Wetterneck, \& Flessner, 2006). In a multiple baseline with four adults diagnosed with OCD (Twohig, Hayes, \& Masuda, 2006a), eight weekly 1-hr sessions of ACT without in-session exposure had a significant impact on OCD severity, depression, and anxiety. OCD severity scores moved from the clinical to the nonclinical range, and results showed near zero levels of compulsions by the end of treatment, with results maintained at follow-up. ACT processes were functionally related to outcomes. To date, however, there is no published randomized controlled trial on ACT for OCD.

In this study, we tested whether ACT for OCD can alter psychological flexibility and effectively reduce OCD. Although formal in-session exposure procedures fit within an ACT approach to OCD, they were not included in this investigation in order to determine whether ACT procedures were effective without these methods of known impact. Outside of treatment sessions, clients were not asked to confront feared stimuli and stay in their presence until a reduction in fear occurred, but instead were asked to practice ACT skills when they encountered feared stimuli as part of everyday functioning. Because the focus of the present study was on acceptability, efficiency, and applicability, the protocol tested was deliberately short, with eight 1 -hr sessions, and included all subtypes of OCD. Very few additional exclusion criteria were applied: Only individuals with active psychosis or a cognitive deficit that would make it difficult to participate (e.g., autism) were excluded. Finally, given the limited data on the effectiveness of ACT for OCD, as a first step in establishing efficacy, it seemed prudent to compare ACT with a credible comparison condition that controls for many important factors such as contact with therapist, expectation of change, effects of measurement, passage of time, and other nonspecific factors. Progressive relaxation training was chosen given its successful use as a credible control condition in previous OCD outcome work (e.g., Fals-Stewart, Marcks, \& Schafer, 1993) and given its moderate effectiveness as a treatment for other anxiety disorders (Manzoni, Pagnini, Castelnuovo, \& Molinari, 2008).

\section{Method}

\section{Participants}

Participants were recruited in two western U.S. states through postings, announcements, advertisements, and referrals from health professionals from 2006 to 2009. To be included in the study, participants needed to meet criteria for OCD on the Structured Clinical Interview for DSM-IV (SCID; First, Spitzer, Gibbon, \& Williams, 2002) and be 18 years of age or older. Participants could not have initiated new psychotropic medications or changed dosages within 30 days of beginning the study, be receiving psychotherapy elsewhere or have ceased psychotherapy less than 30 days prior to enrolling in the study, or be diagnosed with a current psychotic disorder or have an organic mental disorder that would severely interfere with their ability to participate.

A participant flowchart is shown in Figure 1. Two hundred twenty-two individuals contacted the researchers with questions, 116 of whom did not participate in the study. Of the 116 who did not participate, $56(48 \%)$ did not qualify for the study during the initial phone screening (e.g., called to learn about OCD, called for someone else, were too young to participate, or called regarding symptoms for a related disorder such as trichotillomania) and 60 $(52 \%)$ were invited to participate in the intake to determine their formal eligibility for the study but did not do so (e.g., they did not attend and were unable to be contacted, said that they wished to seek psychotropic medications or desired to change them, were not interested in psychotherapy, or lived more than $2 \mathrm{hr}$ away from the study location and did not wish to commute). Of the 106 who participated in the intake sessions, 80 met criteria to participate in the investigation, and 79 agreed to participate after qualifying. The one eligible participant who refused participation (before random assignment) cited scheduling problems as the reason. Treatment assignment was made by a random sequence of concealed slips, opened following the agreement to participate by a person other than the assessors (41 ACT, 38 PRT). No participants overtly 


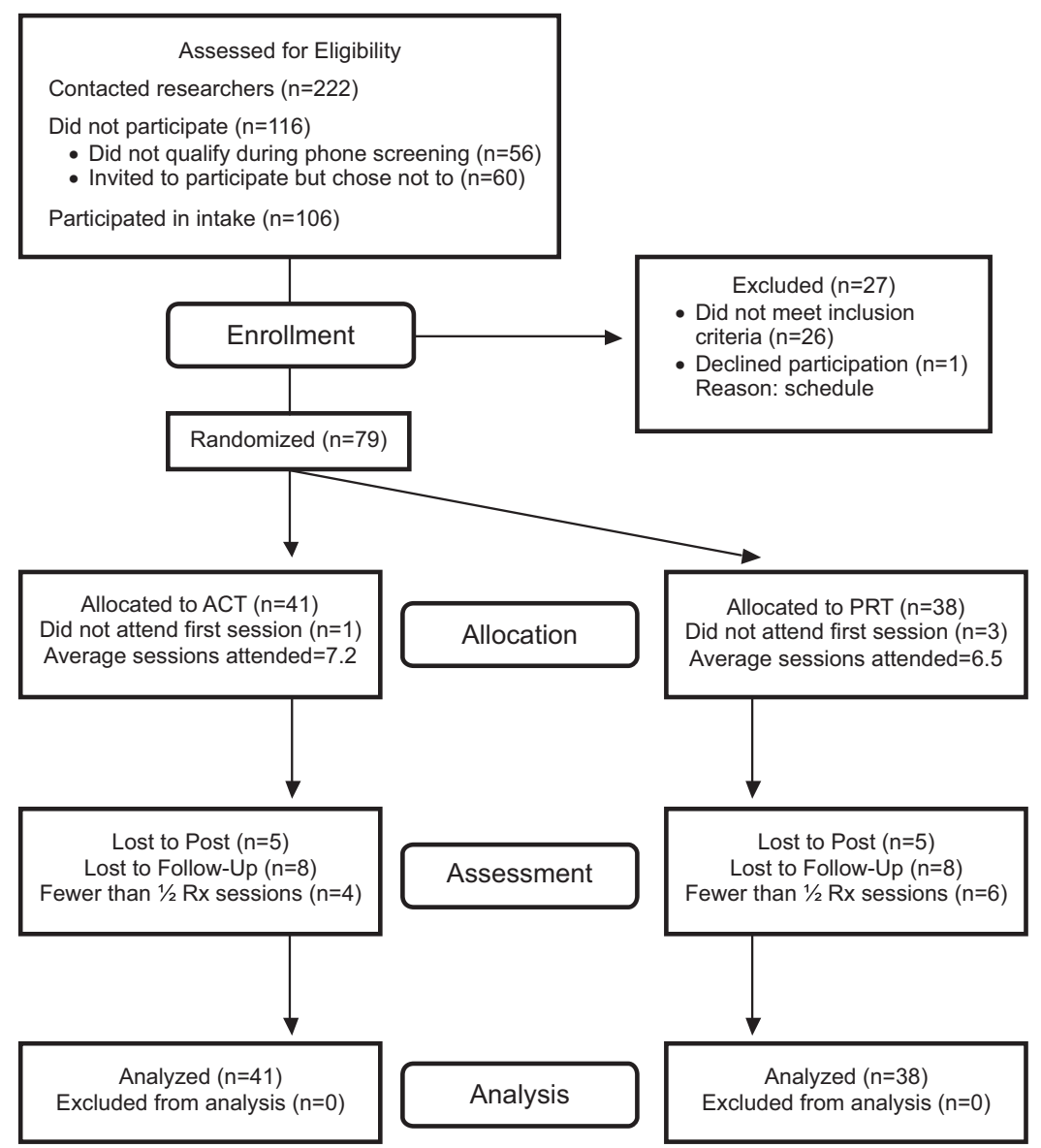

Figure 1. Participant flow through the study. ACT $=$ acceptance and commitment therapy; PRT $=$ progressive relaxation training.

refused participation after random assignment. An number of 38 per condition was required for power of .8 to detect the average between group effect size $(d=0.66)$ in the existing ACT literature across all diagnoses (Hayes et al., 2006). All randomized participants were included in the main analyses. The rationale for the assigned treatment modality was covered extensively in the first treatment session. Participants were not informed of the details of the alternate treatment condition until the end of follow-up, at which time they were given the option to engage in eight sessions of the treatment they did not receive.

Demographic and diagnostic characteristics of the sample were assessed via a self-report questionnaire and the SCID. There were slightly more women in the study $(61 \%)$; average age of participants was 37 years $(S D=15.5$; range: $18-67)$. The sample was 88.6\% Caucasian (1\% African American, 2.5\% Asian American, $5 \%$ Latin American, and $2.5 \%$ Native American). The average educational level was 14.9 years $(S D=2.0)$ of schooling, with first grade being the first year and senior year of high school being the 12 th. Thirty-three percent of the sample reported being married, and 50\% reported being unmarried, with $11 \%$ divorced, $1.3 \%$ separated, and $2.5 \%$ widowed. Participants reported experiencing OCD symptoms for an average of 20.5 years $(S D=17.1$; range: 1-62) and that their OCD symptomatology had been a significant problem for 14.5 years $(S D=14.0$; range: $1-57)$. Sixty-three percent of the participants had received previous formal treatment (psychotropic medications or psychotherapy) for OCD. At intake, $40 \%$ were taking psychotropic medications (34\% in ACT, $44 \%$ in PRT). The frequencies of psychotropic medications per condition were as follows: antidepressants, 14 ACT and 16 PRT; benzodiazepines, four ACT and two PRT; atypical antipsychotics, two ACT and three PRT; anticonvulsants, one ACT and one PRT; and psychostimulants, one ACT and two PRT. The frequencies of the total number of psychotropic medications per individual per condition were as follows: one medication, eight ACT and 11 PRT; two medications, four ACT and five PRT; three medications, two ACT and zero PRT; and four medications, zero ACT and one PRT. There were no statistically significant differences between treatment conditions on any of these demographic factors.

According to the SCID, all participants met criteria for OCD as their primary diagnosis, with $51 \%$ receiving one or greater additional diagnoses. The frequency of co-occurring diagnosis per condition were as follows: mood disorders, 12 ACT and 12 PRT; anxiety disorder besides OCD, nine ACT and 13 PRT; and substance dependence, two ACT and two PRT. One participant met criteria for bulimia nervosa and one for hypochondriasis in the PRT condition, and one met criteria for adjustment disorder in 
ACT. The frequencies of total number of co-occurring diagnoses per individual per condition were as follows: one, 14 ACT and 7 PRT; two, four ACT and eight PRT; and three, one ACT and two PRT.

OCD subtypes for the sample were assessed via the Yale-Brown Obsessive Compulsive Scale (Y-BOCS; Goodman et al., 1989). Only $25 \%$ of the participants presented with one subtype of OCD; the remaining $75 \%$ presented with two or greater subtypes. It is not possible to rate severity of subtypes from these data. With no more than the three highest rated OCD subtypes counted for each person from the Y-BOCS, 19 ACT and 18 PRT presented with contamination, $27 \mathrm{ACT}$ and 15 PRT presented with symmetry/exactness, five ACT and four PRT presented with safety/harm, 18 ACT and 16 PRT presented with doubt/checking, 12 ACT and eight PRT presented with religious/sexual/scrupulosity, and nine ACT and 16 PRT presented with hoarding. On the basis of Fisher's exact test, none of these differences in co-occurring disorders or subtypes of OCD were significantly different $(p<.05)$ between conditions except that those in the ACT condition had more concern with symmetry/exactness $(p=.03)$.

\section{Therapists, Assessors, and Setting}

The primary supervisors were Steven C. Hayes, Holly HazlettStevens, and Michael P. Twohig. Each has written books relevant to areas of this study: ACT (Hayes et al., 1999), relaxation training (Bernstein, Borkovec, \& Hazlett-Stevens, 2000), and acceptancebased approaches to OCD spectrum disorders (Woods \& Twohig, 2008). All therapists $(N$ : ACT $=2, \operatorname{PRT}=4)$ and assessors $(N=$ 4) were advanced graduate students in clinical psychology. All assessors completed all assessments (pretreatment, posttreatment, and follow-up) on the same participants. ACT therapists were trained and supervised by Hayes; PRT therapists were trained and supervised by Hazlett-Stevens (32 cases) or Twohig (six cases), who was trained by Hazlett-Stevens in PRT. Assessors were supervised by Hayes or Twohig and were blind to treatment assignment throughout. All assessments and therapy sessions occurred in standard therapy rooms that were equipped with video-recording equipment.

\section{Diagnosis}

The SCID was used to diagnose Axis I disorders. The SCID is considered the gold standard in psychiatric diagnosis and has shown largely consistent diagnostic reliability across studies (Segal, Hersen, $\&$ Van Hasselt, 1994). In general, $\kappa>.70$ has been found for most clinical disorders, and validity is generally noted based on the SCID's similarity to the Diagnostic and Statistic Manual of Mental Disorders (Summerfeldt \& Antony, 2004).

\section{Outcome Measures}

Y-BOCS. The primary outcome was OCD severity as measured by the Y-BOCS (Goodman et al., 1989). The Y-BOCS is an assessor-rated measure of symptom severity for OCD. Total scores on the Y-BOCS range from 0 to 40 . The Y-BOCS has good interrater reliability for the total score $(r \mathrm{~s}=.80-.97)$ and 2-week test-retest reliability between .81 and .97 . In the present trial, Cronbach's $\alpha$ at pretreatment was .79 .
Beck Depression Inventory-II (BDI-II). Because depression is commonly associated with OCD, the BDI-II (Beck, Steer, \& Brown, 1996) was used as a secondary outcome measure. The BDI-II has shown good internal consistency and high test-retest reliability. In the present trial, $\alpha=.90$.

Quality of Life Scale. The Quality of Life Scale (Burckhardt, Woods, Schultz, \& Ziebarth, 1989) measures how satisfied people are with the quality of their lives. It has been found to be internally consistent $(\alpha \mathrm{s}=.89-.92)$, with good temporal stability ( $r \mathrm{~s}=$ $.78-.84$ over 3 weeks; Burckhardt et al., 1989). In the present trial, $\alpha=.89$.

\section{Measures of Treatment Process}

Acceptance and Action Questionnaire. The 16-item Acceptance and Action Questionnaire (Hayes et al., 2004) was used to measure psychological flexibility. Questions are rated on a 7-point Likert-type scale. Higher scores reflect greater experiential willingness and ability to act in the presence of difficult thoughts and feelings. Sample items include "It's OK to feel depressed or anxious" and "It is unnecessary for me to learn to control my feelings in order to handle my life well." The Acceptance and Action Questionnaire has good convergent and discriminant validity, and test-retest reliability after a 4-month period was $r=.64$ (Hayes et al., 2006, 2004). In the present trial, $\alpha=.74$.

Thought Action Fusion Scale. The Thought Action Fusion Scale (Shafran, Thordarson, \& Rachman, 1996) contains 19 items designed to measure how much participants equate thought and action. Items are rated from 0 (strongly disagree) to 4 (strongly agree). It has two subscales: (a) having a thought makes an event more likely to happen (likelihood thought-action fusion) and (b) having the thought is the same as engaging in the action (moral thought-action fusion). Mean scores of an obsessional sample (diagnosed with OCD or high scores on an OCD severity measure) on the likelihood subscale were 9.18 , and 3.12 for the community sample, and scores on the moral subscale were 20.03 for the obsessional sample and 12.74 for the community sample. Threemonth test-retest reliability for the total score were $r=.52$, and it shows construct validity with other measures (Rassin, Merckelbach, Muris, \& Schmidt, 2001). In the present trial, $\alpha=.92$; thought-action fusion moral subscale, $\alpha=.91$; thought-action fusion likelihood subscale, $\alpha=.95$.

Thought Control Questionnaire. The Thought Control Questionnaire (Wells \& Davies, 1994) is a 30-item self-report measure that assesses the use of thought control strategies. An example item includes, "When I experience an unpleasant/ unwanted thought I think pleasant thoughts instead." Test-retest reliability is good $(r=.83)$. This is a widely used measure of this construct for obsessive-compulsive and anxiety-related disorders and has been shown to change as a result of cognitive behavioral interventions (e.g., Reynolds \& Wells, 1999). In the present trial, $\alpha=.82$.

\section{Measures of Treatment Credibility and Acceptability}

Treatment credibility measure. Treatment credibility was measured immediately after the initial therapy session with five questions from the Credibility/Expectancy Questionnaire (Devilly $\&$ Borkovec, 2000) worded for the treatment of OCD. An example 
item is "How logical does this type of treatment seem to you for helping people reduce their obsessive-compulsive disorder?" Questions were rated on a 0- to 8-point Likert scale. Participants were asked to complete the measure and seal it in an envelope that would not be opened until they had completed the study. This was done to preserve the confidentiality of this information during treatment.

Treatment Evaluation Inventory-Short Form. Acceptability of treatment was assessed only at posttreatment with the acceptability factor of the Treatment Evaluation Inventory-Short Form (Kelley, Heffer, Gresham, \& Elliott, 1989), with two questions left out that pertain only to family interventions. A sample item is "I liked the procedures used in this treatment." In the present trial, $\alpha=.94$.

\section{Assessment Occasions}

Participants were assessed 1 week prior to treatment (pretreatment). In addition to providing consent for participation and demographic information, participants completed the SCID and all measures. These were repeated 1 week (posttreatment) and 3 months (follow-up) after the final treatment session except as noted. Weekly outcome and process measures were taken during treatment (these will be addressed in a subsequent publication).

\section{Treatment Procedures}

Participants attended 11 total sessions: an intake session, eight 1-hr weekly treatment sessions, a posttreatment assessment session, and a 3-month follow-up assessment session. Following the initial intake, participants were randomly assigned to either the ACT for OCD or the PRT for OCD condition. A brief description of the interventions follows.

ACT for OCD. The protocol used in the ACT for OCD investigation is the same as that used by Twohig et al. (2006a). ${ }^{1}$ Although book-length presentations of ACT for anxiety are available (e.g., Eifert \& Forsyth, 2005; Woods \& Twohig, 2008), ACT is still unfamiliar enough that an extended characterization of the protocol seems warranted. Sessions followed the same essential pattern: Homework and events over the past week were discussed, the material from the previous session was reviewed and new material was presented, and new homework was assigned and behavioral commitment exercises were agreed upon (behavioral commitments began at Session 3). Behavioral commitment exercises involved commitments to engage in valued-directed behavior that was both important and difficult. Examples of behavioral commitments included participating in church services, playing sports, eating lunch with others, and spending time with family. These tasks were difficult because of their OCD. For example, the person playing sports might get dirty and have fears of contamination; the person going to church might disappear into scrupulous ruminations; the person eating lunch with others might struggle with unwanted sexual images of those near him; the person spending time with family might contract an urge to clean the house instead. The goal of these commitments was not to reduce obsessions or anxiety levels; rather they were presented as opportunities to engage in important life values while practicing ACT skills (e.g., acceptance, defusion, present moment awareness) if private experiences arose that might interfere with valued actions. For example, a client playing sports might mindfully notice thoughts of contamination as if watching an external object, spend some moments feeling where anxiety arises in his or her body, and then shift attention toward playing the sporting activity with gusto. These behavioral commitments were established as specific activities for specified periods, without regard to the nature or intensity of the participant's obsessions or anxiety.

Session 1 began by examining in detail the participant's obsessions and compulsions, introducing the treatment, and establishing a verbal therapeutic contract. Next, therapists helped participants identify and distinguish between obsessions and compulsions. The participant was shown how one could occur without the other but that in the participant's life they usually occurred together. The participant was asked what he or she had done to decrease the obsession, with particular attention paid to which strategies had worked to reduce obsessions and which had not. The purpose of this "creative hopelessness" phase of treatment was to note that seemingly the only way to obtain relief from the obsession was to engage in a compulsion, but that this strategy only worked for brief periods, and this process generally pulled the participant into a greater struggle with the obsessions.

Session 2 focused on the "Man in the Hole" metaphor (Hayes et al., 1999, p. 101), which demonstrates the ultimate ineffectiveness of attempts to regulate inner experiences such as obsessions. The metaphor describes the participant falling in a hole (which represents the obsession) with only a shovel to get out (methods for reducing the obsession). The metaphor illustrates how the participant's attempts to dig out of the hole (representing attempts to reduce or control the obsession) are unsuccessful and actually make the hole larger (the paradox of how struggling with one's obsessions can make them more central, aversive, and connected with diverse situations or actions). Being stuck in a hole served as a metaphor for how such strategies often result in high personal costs such as reduced engagement in social and occupational activities. The intended function of the metaphor, and its extension to the participant's life, was to reduce the focus on reducing obsessions and increase awareness of the difficulty in controlling it.

Sessions 3 and 4 generally focused on illustrating how attempts to control obsessions might be exacerbating OCD rather than ameliorating it and that allowing the obsessions to occur might be more useful in the long term. This involved exercises aimed at illustrating the limitations of control when aimed at inner experiences, such as trying not to think of something, such as chocolate cake (Hayes et al., 1999, p. 124), or not to get nervous when hooked to a polygraph machine (Hayes et al., 1999, p. 123). These exercises were designed to help the participant experience the difference between an obsession (an uncontrollable inner experience) and a compulsion (a controllable public behavior), hopefully shifting the focus from decreasing the obsession to decreasing compulsive behaviors. The "Two Scales Metaphor" (Hayes et al., 1999, p. 133) was discussed to illustrate the possible benefits of acceptance of obsessions and other inner experiences such as anxiety over attempting to control them. Scale 1 represented the severity of the obsession, and it was shown that this scale is very difficult to regulate. Scale 2 represented willingness to experience

\footnotetext{
${ }^{1}$ The protocols are available at http://www.contextualpsychology.org or from the first author.
} 
the obsession, and it was shown how this scale could be regulated. The participant was told that treatment focused on increasing Scale 2 (willingness) while learning not to address Scale 1 (obsessions). It was presented that being willing would not necessarily decrease the obsession or anxiety, but that being unwilling certainly increases them or makes them more prominent. Willingness therefore means that the participant can allow the obsession and feelings of anxiety to rise and fall as they will, while the participant engages in important life activities. Attempts to control obsessions, on the other hand, can paradoxically increase their frequency, intensity, and capacity to control behavior.

Sessions 5 and 6 focused on changing the psychological function of the obsession from something threatening to simply another cognitive event. Defusion exercises, contact with the present moment or mindfulness exercises, and self as context work were employed to this end. Examples of defusion exercises were speaking a thought in a funny voice and treating the obsessions as passengers on a bus where the participant is the bus driver. This metaphor was used to illustrate that the passengers had been allowed to take control of the bus rather than the driver (the participant's response to obsessions cost control over life direction) and that control of the bus could go back to the driver if the driver was willing to allow the passengers to come along for the ride. The participant was helped to recognize that the passengers grew more unruly (the obsessions felt more intense) as he or she began driving the bus in chosen life directions (toward values) but that he or she had ultimate control over the bus's movement (Hayes et al. 1999, p. 157).

Contact with the present moment involved helping the participant flexibly attend to the world more directly, rather than the world as constructed by the evaluative language processes. This process is very similar and contains many of the same principles as other acceptance- or mindfulness-based therapies. Contact with the present moment was fostered by experiential exercises such as the "Leaves on a Stream" (Hayes et al., 1999, p. 158), wherein thoughts and other experiences can be witnessed as events floating down a stream. The goal of such exercises is to practice noticing experiences in the present moment without holding onto (inflexibly attending to or struggling with) any one thought or feelingjust observing what occurs.

Self as context work helps the participant experience a sense of self as more than his or her inner experiences - as an observer of them. Participants are helped to see inner experiences as simply transient events to be observed rather than as identifying personal characteristics. The "Chessboard Metaphor" (Hayes et al., 1999, p. 190) was used, in which the participant is described as the chessboard and the pieces are the obsessions, to help the participant see that the obsessions could exist without causing harm to the participant-just as the chessboard can exist without being harmed by the pieces.

Sessions 7 and 8 involved discussing each participant's values and increased behavioral commitments to live more consistently with them. Values were assessed in session with the Valued Living Questionnaire (Wilson, Sandoz, Kitchens, \& Roberts, 2010). The questionnaire assessed the discrepancy between the importance of several personal values domains including family, occupation, and recreation and one's success at living consistent with these values. For many participants, engaging in compulsions had been a barrier to living in accordance with one or more valued domains. Based on the responses to these questions, larger behavioral commitments were planned that involved following one's values and practicing willingness to experience obsessions as one did so. Finally, the therapist helped the participant see how to prevent relapse by practicing acceptance, present moment, defusion, and self as context exercises while engaging in increasingly consistent patterns of values-directed action.

PRT for OCD. An abbreviated version of PRT based on Bernstein et al. (2000) was used in this study. It involved learning to tense and relax groups of muscles beginning with a larger number of smaller groups (in this case 16) and then proceeding in steps to a smaller number of larger groups (seven groups and four); finally, relaxation was taught by recall and counting alone. PRT sessions followed a similar pattern as the ACT condition: Events since the last session and homework were reviewed, the material from the previous session was reviewed and new material was presented, and new homework was assigned. In Session 1, anxiety was presented as a precipitating factor for obsessions, and PRT as a way to help manage anxiety. Sessions 2-4 involved engaging in 16-muscle group relaxation, twice each session. Participants were asked to engage in the procedure at least twice a day at home. In Sessions 5 and 6, the focus was on seven-muscle group relaxation; Session 7 focused on four-muscle group relaxation. Recall (bringing on the relaxed state by remembering the experience of being relaxed) was also trained and practiced. Session 8 reviewed the four-muscle group method and recall, and trained a cuing procedure based on counting.

\section{Reliability of OCD Diagnosis and Y-BOCS Scores}

All assessment sessions were recorded. Twenty percent of the initial diagnostic interviews were independently coded by a second rater; there was $100 \%$ agreement on diagnosis and nondiagnosis of OCD. A random selection of $10 \%$ of the Y-BOCS tapes from pretreatment, posttreatment, and follow-up were rated by a second rater. Overall agreement was .90 (95\% CI [.87, .92]) with a two-way mixed intraclass correlation coefficient, suggesting very high agreement on Y-BOCS scores between the original assessor scores and the additional rater scores taken from the tapes.

\section{Treatment Integrity}

ACT condition. A coding system that was successfully employed in a previous study on ACT for OCD (Twohig et al., 2006a) was used in the current study to rate treatment integrity. Twenty percent of each ACT therapist's sessions (distributed evenly across all eight treatment sessions) was rated. In addition to rating the overall adherence to the manual, overall therapist competence, and the degree to which therapists assessed the participant's functioning, the coding system rated the frequency and depth of coverage of major components of the ACT model on a 5-point scale. Each rating scale and item includes anchors and examples. Nonadherent items were also coded including challenging cognitions, use of a cognitive therapy rationale, supporting the idea that thoughts and feelings lead to action, using avoidant change strategies, and using ERP within session or suggesting its use outside of session. ERP exercises were generally defined as purposefully exposing oneself to a feared stimulus, for an extended duration, to produce anxiety and obsessions, and staying in that situation until a reduction in 
anxiety or obsessions occurs. Three independent raters completed the coding with $20 \%$ overlap in order to calculate reliability using two-way mixed intraclass correlation coefficients. An advanced clinical graduate student trained in ACT served as a master rater. Interrater reliability across all domains assessed for integrity (described in detail below) was .94 (95\% CI $[.90, .96])$ between the master rater and Rater 1 and .80 (95\% CI $[.63, .90])$ between the master rater and Rater 2.

Scores of 1 on the rating system indicate that the variable was never explicitly covered; 2 , that the variable occurred at least once and not in an in-depth manner; 3 , that the variable occurred several times and was covered at least once in a moderately in-depth manner; 4, that the variable occurred with relatively high frequency and was addressed in a moderately in depth manner; and 5, that the variable occurred with high frequency and was covered in a very in-depth manner. No nonadherent items were noted in any of the coded sessions. Overall adherence to the manual and overall competence were highly rated $(M=4.56, S D=0.54$, and $M=$ $4.67, S D=0.48$, respectively). These ratings are the primary adherence and competence scores because particular processes varied from session to session. In order to characterize the sessions more completely, however, it is helpful to examine specific item scores for ACT processes. Means for ACT components over the eight sessions were as follows: creative hopelessness/workability, 3.09 ( $S D=1.47)$; defusion, 2.82 ( $S D=1.26)$; willingness/ acceptance, $2.91(S D=1.41)$; values and goals, $2.68(S D=1.15)$; committed action, $1.74(S D=1.03)$; and assessment of participant's symptoms and overall functioning, $3.12(S D=0.95)$; indicating that for most processes the "variable occurred several times and was addressed at least once by the therapist in a moderately in-depth manner" in each session on average (the manual definition of a score of 3). The focus of specific sessions can be examined by considering the highest mean item rating for each session, which was as follows: Session 1: general assessment, 4.38 $(S D=0.74)$; Session 2: creative hopelessness/workability, 5.00 $(S D=0.00)$; Session 3: creative hopelessness/workability, 4.38 $(S D=0.92)$; Session 4: willingness/acceptance, $3.33(S D=0.52)$, and defusion, 3.33 (1.03); Session 5: defusion, 4.13 (0.64); Session 6: defusion, 3.86 (1.07); Session 7: values and goals, 3.83 ( $S D=$ $0.75)$; Session 8: values and goals, $3.67(S D=1.03)$.

PRT condition. A PRT integrity coding manual was developed with the same framework as the ACT integrity coding manual (the rating scale, percentage of tapes examined, and reliability checks were the same across the two conditions) and included components based upon the PRT therapy manual. Rated nonadherent items were ERP and ACT processes (creative hopelessness/ workability, defusion, acceptance, values, and committed action). An advanced clinical graduate student trained in PRT served as a master rater. Interrater reliability across all domains assessed for integrity (described in detail below) between the master rater and Rater 1 was .98 (95\% CI $[.93, .98])$ and between the master rater and Rater 2 was .95 (95\% CI [.82, .96]).

On a 5-point scale, PRT therapists' overall adherence to the manual and overall competence were highly rated $(M=4.60$, $S D=0.73$, and $M=4.65, S D=0.57$, respectively). The frequency of nonadherent items was very low but occurred in the areas of acceptance $(M=1.07, S D=0.26)$, creative hopelessness/ workability $(M=1.02, S D=0.15)$, and $\operatorname{ERP}(M=1.11, S D=$ 0.39). Means for PRT components over the eight sessions were as follows: PRT rationale, $2.74(S D=1.26)$; relaxation procedure, $3.56(S D=1.31)$; homework practice, $2.77(S D=0.84)$; and assessment of participant's symptoms and overall functioning, $3.44(S D=0.79)$. Examining the data session by session showed that the relaxation procedure item was scored highest of all items in all sessions (range of the session means for that item: 3.2-4.5) except the first session in which the PRT rationale was highest: $4.67(S D=0.82)$.

\section{Results}

Table 1 shows the obtained means for all outcome measures for the two conditions for each measurement occasion. We compared pretreatment differences on parametric measures using $t$ tests; other measures (or those violating homoscedasticity) were tested

Table 1

Means and Standard Deviations for All Outcome Measures for the Two Conditions for Each Measurement Occasion

\begin{tabular}{|c|c|c|c|c|c|c|c|c|c|c|c|c|}
\hline \multirow[b]{3}{*}{ Measure } & \multicolumn{6}{|c|}{$\mathrm{ACT}$} & \multicolumn{6}{|c|}{ PRT } \\
\hline & \multicolumn{2}{|c|}{$\begin{array}{l}\text { Baseline } \\
(N=41)\end{array}$} & \multicolumn{2}{|c|}{$\begin{array}{l}\text { Posttreatment } \\
\quad(N=36)\end{array}$} & \multicolumn{2}{|c|}{$\begin{array}{l}\text { Follow-up } \\
(N=33)\end{array}$} & \multicolumn{2}{|c|}{$\begin{array}{l}\text { Baseline } \\
(N=38)\end{array}$} & \multicolumn{2}{|c|}{$\begin{array}{l}\text { Posttreatment } \\
\quad(N=33)\end{array}$} & \multicolumn{2}{|c|}{$\begin{array}{l}\text { Follow-up } \\
(N=31)\end{array}$} \\
\hline & $M$ & $S D$ & $M$ & $S D$ & $M$ & $S D$ & $M$ & $S D$ & $M$ & $S D$ & $M$ & $S D$ \\
\hline \multicolumn{13}{|l|}{ Outcome } \\
\hline Y-BOCS & 24.22 & 4.80 & 12.76 & 8.35 & 11.79 & 8.97 & 25.40 & 5.26 & 18.67 & 5.68 & 16.23 & 7.46 \\
\hline BDI-II & 18.09 & 11.83 & 8.50 & 10.99 & 8.43 & 10.13 & 17.89 & 8.39 & $13.69^{\mathrm{a}}$ & 9.83 & 10.89 & 8.06 \\
\hline QOLS & 72.49 & 15.00 & 82.18 & 14.86 & 82.03 & 14.58 & 69.58 & 14.35 & $73.59^{\mathrm{a}}$ & 12.42 & 73.19 & 17.01 \\
\hline \multicolumn{13}{|l|}{ Process } \\
\hline AAQ-16 & 59.76 & 11.36 & 73.69 & 13.22 & 73.37 & 14.44 & 57.16 & 11.67 & $63.81^{\mathrm{a}}$ & 7.69 & $67.13^{\mathrm{b}}$ & 10.74 \\
\hline TCQ & 65.54 & 12.23 & 56.11 & 10.76 & 56.74 & 9.66 & 65.04 & 8.04 & $61.80^{\mathrm{a}}$ & 8.44 & 60.26 & 6.73 \\
\hline TAF-Morality & 21.46 & 11.17 & 12.72 & 9.89 & 14.14 & 10.58 & 18.47 & 10.38 & $17.94^{\mathrm{a}}$ & 9.49 & 18.13 & 9.53 \\
\hline TAF-Likelihood & 9.76 & 8.21 & 5.47 & 6.47 & 6.90 & 7.56 & 7.76 & 7.66 & $6.22^{\mathrm{a}}$ & 6.63 & 6.00 & 5.81 \\
\hline
\end{tabular}

Note. $\quad \mathrm{ACT}=$ acceptance and commitment therapy; PRT $=$ progressive relaxation training; Y-BOCS $=$ Yale-Brown Obsessive Compulsive Scale; BDI-II = Beck Depression Inventory-II; QOLS = Quality of Life Scale; AAQ-16 = Acceptance and Action Questionnaire (16-item version); TCQ = Thought Control Questionnaire; TAF = Thought Action Fusion.

${ }^{\mathrm{a}} N=32 .{ }^{\mathrm{b}} N=30$. 
with a Mann-Whitney $U$ test. There were no pretreatment differences $(p<.05)$ on any measure.

\section{Dropout and Dose}

In the present study no participants overtly refused treatment assignment; one of the 41 participants in the ACT condition $(2.4 \%)$ and three of the 38 participants in the PRT condition $(7.9 \%)$ did not attend Session 1, indicating very low passive treatment refusal. The rates for minimal participation or passive dropout were similarly low: Four ACT participants $(9.8 \%)$ and five PRT participants $(13.2 \%)$ attended only one or two sessions.

\section{Outcome Analytic Strategy}

Longitudinal data were analyzed with hierarchical linear modeling (HLM) and mixed model repeated measures (MMRM) approaches and an intent-to-treat sample containing all randomized participants (Hedeker \& Gibbons, 2006). HLM analyses were conducted on all measures. Measurement occasion was used as a continuous temporal variable, with pretreatment set as Occasion 0. HLM analyses were first conducted assuming a random intercept and slope and allowing them to be correlated, followed by analyses assuming they were uncorrelated and a random intercept analysis. Final analyses relied on the simplest variance-covariance structure not significantly different from the most complex as determined by comparison of models through the restricted log-likelihood. If the HLM analyses did not fully converge (indicated by the Hessian matrix not positive definite) or the HLM model fit less well than MMRM (comparing an HLM with unstructured covariance with an MMRM analysis with compound symmetry covariance assumptions using the significance of restricted log-likelihood values), then an MMRM analysis was used that treated time as a categorical factor. Analyses first were conducted with unstructured covariance assumptions, estimating a separate variance for each time point and separate covariance for each distinct pair of time points, followed by more restrictive assumptions (using compound symmetry, Toeplitz, and heterogeneous compound symmetry covariance structures). The model with the fewest covariance parameters was used provided there was no significant difference in fit compared with an unstructured covariance model as determined by comparison of models through the restricted log-likelihood.

Denominator degrees of freedom for fixed-effects test statistics were based on the Satterthwaite approximation. Effect sizes for MMRM outcomes were calculated as specified by Wackerly, Mendenhall, and Scheaffer (2008) and for HLM by examining end-point outcomes as suggested by Feingold (2009). Effect sizes were calculated with Cohen's (1988) cutoffs (small $\geq 0.2$, medium $\geq 0.5$, large $\geq 0.8$ ).

\section{Primary Outcome: Y-BOCS}

The overall pattern of results from the Y-BOCS is shown in Figure 2. Because the slopes per se are included in the text, the figure shows the adjusted means from an MMRM analysis with unstructured covariance. An HLM model assuming that slopes and intercepts were uncorrelated best fit the data. There was a significant effect for time, $F(1,80.64)=115.54, p<.001$; no effect for condition, $F(1,92.98)=1.16, p=.28$; and a significant interac-

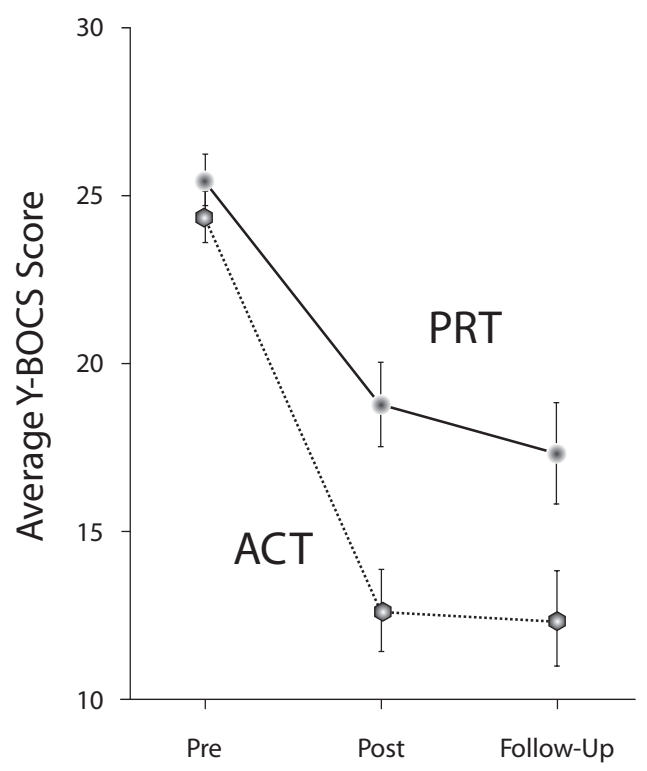

Figure 2. Yale-Brown Obsessive Compulsive Scale (Y-BOCS) adjusted means and standard errors (taken from a mixed model repeated measures with unstructured variance) for each condition at the pretreatment, posttreatment, and follow-up assessment periods. Slopes from the final hierarchical linear modeling analysis are indicated in the text. ACT $=$ acceptance and commitment therapy; PRT $=$ progressive relaxation training.

tion between condition and time, $F(1,80.64)=5.15, p=.026$. Participants in the ACT (estimate $=-1.22, S E=0.13$ ) and PRT (estimate $=-0.79, S E=0.13$ ) conditions showed significantly improving slopes, $t(80.90)=-9.40, p<.001,95 \%$ CI $[-1.47$, $-0.96]$, and $t(80.40)=-5.88, p<.001,95 \%$ CI $[-1.06,-0.52]$, respectively, but the ACT condition produced a large and significant difference in rate of improvement (estimate $=0.42, S E=$ $0.19), t(80.64)=2.27, p=.026,95 \%$ CI [0.05, 0.80], end-point outcome effect size $=0.84$.

The Jacobson methodology (Jacobson, Follette, \& Revenstorf, 1984; Jacobson \& Truax, 1991) was used to analyze the clinical significance of the obtained Y-BOCS scores. A change of 6.39 on the Y-BOCS was considered reliable. Method A for determining the cutoff score for clinically significant change subsequent to a reliable change yielded a cutoff of 14 at posttreatment or followup, which is the same cutoff as a recent meta-analysis evaluating ERP and ERP-CT for OCD (Fisher \& Wells, 2005). All refusals, dropouts, and missing data were scored as unimproved and included in the denominator, providing a full intent-to-treat analysis. The two conditions differed at posttreatment (ACT: 19 of 41; PRT: 5 of 38), $\chi^{2}(1)=10.27, p=.002$ (two-tailed), effect size $=0.77$, and follow-up (ACT: 19 of 41; PRT: 7 of 38), $\chi^{2}(1)=6.96, p<$ .009 (two-tailed), effect size $=0.62$. To deal with missing data, researchers frequently report these figures using the last value carried forward, or including only completers instead of assuming that all missing data are negative. A more sophisticated approach to missing data is to use imputed values from the person-specific growth curves from the HLM analysis. With these values, the two conditions again differed at posttreatment (ACT: 23 of 41; PRT: 7 of 38), $\chi^{2}(1)=10.30, p<.002$ (two-tailed), effect size $=0.77$, 
and follow-up (ACT: 27 of 41; PRT: 6 of 38), $\chi^{2}(1)=18.24, p<$ .001 (two-tailed), effect size $=1.10$.

\section{Secondary Outcomes}

Depression. Depression scores were analyzed for those participants who were at least mildly depressed at pretreatment based on a BDI-II cutoff score of 13 . Seventy percent of the sample $(N$ : $\mathrm{ACT}=27, \mathrm{PRT}=28)$ met that criterion. An MMRM analysis with a heterogeneous compound symmetry structure best fit the data and revealed a nonsignificant effect for treatment condition, $F(1,55.32)=0.35, p=.56$, but significant effects for time, $F(2$, $69.63)=44.14, p<.001$, and the interaction between condition and time, $F(2,69.63)=5.61, p=.006$. The interaction occurred because ACT participants with at least a mild level of depression improved significantly more on the BDI-II than PRT participants from pre- to posttreatment (estimated $M_{\text {diff }}=-8.10, S E=2.51$ ), $t(59.65)=-3.23, p=.002,95 \%$ CI [-13.12, -3.09], effect size $=0.96$, and from pretreatment to follow-up (estimated $\left.M_{\text {diff }}=-4.87, S E=2.37\right), t(62.82)=-2.06, p=.044,95 \% \mathrm{CI}$ $[-9.60,-0.14]$, effect size $=0.63$. Within-condition pre- to posttreatment and pretreatment to follow-up changes were significant: Effect sizes were respectively 0.5 and 0.93 in the PRT condition and 1.40 and 1.52 in the ACT condition.

Quality of life. An MMRM analysis with unstructured covariance best fit the data and revealed a nonsignificant effect for condition, $F(1,66.53)=2.41, p=.126$, but a significant effect for time, $F(2,64.62)=11.44, p<.001$, and trend toward an interaction between condition and time, $F(2,64.62)=2.99, p=.057$. There was no difference in improvement between conditions at follow-up (estimated $M_{\text {diff }}=0.18, S E=3.14$ ), $t(63.49)=0.06$, $p=.96,95 \%$ CI $[-6.10,6.46]$, effect size $=0.01$, but there was a trend toward a difference at posttreatment (estimated $M_{\text {diff }}=$ $5.46, S E=2.78), t(70.22)=1.96, p=.054,95 \%$ CI $[-0.09$, $11.00]$, effect size $=0.47$.

\section{Process Results}

Acceptance and Action Questionnaire. An MMRM analysis with an unstructured covariance structure best fit the data and revealed a significant effect for treatment condition, $F(1,69.78)=$ 7.30, $p=.009$; time, $F(2,66.36)=22.14, p<.001$; and their interaction, $F(2,66.35)=5.60, p=.006$. The interaction occurred because ACT participants improved significantly more from preto posttreatment than PRT participants (estimated $M_{\text {diff }}=-7.73$, $S E=3.09), t(70.22)=-2.50, p=.015,95 \%$ CI $[-13.90$, -1.96], effect size $=0.59$, but the two conditions were equivalent from pretreatment to follow-up (estimated $M_{\text {diff }}=-3.11, S E=$ 3.40), $t(67.89)=-0.92, p=.36,95 \%$ CI $[-9.89,3.66]$, effect size $=0.22$. Within-condition pre- to posttreatment and pretreatment to follow-up changes were significant: Effect sizes were respectively 0.47 and 0.64 in the PRT condition and 1.06 and 0.86 in the ACT condition.

Thought Control Questionnaire. An MMRM analysis with an unstructured covariance structure best fit the data and revealed an approaching significant effect for treatment condition, $F(1$, $77.28)=3.18, p=.079$, and a significant effect for time, $F(2$, $70.43)=17.88, p<.001$, and the interaction of condition and time, $F(2,70.43)=5.11, p=.008$. The interaction occurred because ACT participants improved significantly more from preto posttreatment than PRT participants (estimated $M_{\text {diff }}=-6.67$, $S E=2.12), t(72.51)=-3.14, p=.002,95 \%$ CI $[-10.90$, $-2.43]$, effect size $=0.75$. There was a trend toward a difference from pretreatment to follow-up (estimated $M_{\text {diff }}=-4.56, S E=$ 2.59), $t(75.70)=1.77, p=.082,95 \%$ CI [-9.72, 0.59], effect size $=0.42$. Within-condition pre- to posttreatment and pretreatment to follow-up changes were respectively $0.31(p=.071)$ and $0.40(p=.022)$ in the PRT condition and $0.80(p<.001)$ and 0.81 $(p<.001)$ in the ACT condition.

Thought Action Fusion Scale. For the moral subscale, an HLM analysis assuming a random slope and intercept that may be correlated revealed a nonsignificant effect for treatment condition, $F(1,76.34)=1.25, p=.27$, and a significant effect for time, $F(1$, $71.72)=16.32, p<.001$, and the interaction of condition and time, $F(1,71.72)=11.39, p=.001$, end-point outcome effect size $=0.73$. ACT participants showed an improving slope (estimate $=-0.86, S E=0.16), t(71.93)=-5.32, p<.001$, but PRT participants did not (estimate $=-0.07, S E=0.17), t(71.52)=$ $-0.46, p=.65$ ). For the likelihood subscale, an HLM analysis assuming only a random intercept revealed a nonsignificant effect for treatment condition, $F(1,117.43)=1.41, p=.24$, and a significant effect for time, $F(1,135.78)=18.94, p<.001$, and a trend toward an interaction of condition and time, $F(1,135.78)=$ $3.33, p=.07$, end-point outcome effect size $=0.31$. ACT participants showed an improving slope (estimate $=-0.41, S E=0.09$ ), $t(135.54)=-4.46, p<.001$, whereas PRT participants showed a trend for one (estimate $=-0.17, S E=0.10), t(136.00)=-1.75$, $p=.082$.

\section{Treatment Credibility, Dose, and Acceptability}

Because the distribution of scores was not normal, treatment credibility was examined nonparametrically with a KolmogorovSmirnov test, which was nonsignificant $(\mathrm{K}-\mathrm{S}=.99, N=71, p=$ $.27)$, indicating that credibility had the same central tendency and distribution in the two conditions. Credibility was not related to follow-up decreases in the Y-BOCS in either the PRT, Spearman's $\rho(31)=-.04, p=.83$, or the ACT condition, Spearman's $\rho(30)=$ $.07, p=.70$. Posttreatment results showed the same pattern.

Because the distribution of scores was not normal, differences between conditions in the number of treatment sessions received were also examined nonparametrically with a KolmogorovSmirnov test, which was nonsignificant $(\mathrm{K}-\mathrm{S}=.98, N=79, p=$ .61). The number of treatment sessions was not related to follow-up decreases in the Y-BOCS in either the PRT, Spearman's $\rho(31)=-.12, p=.54$, or the ACT condition, Spearman's $\rho(33)=$ $-.15, p=.41$. Posttreatment result were similar for the PRT condition, but the number of treatment sessions was correlated with posttreatment decreases in the Y-BOCS in the ACT condition, Spearman's $\rho(33)=-.30, p=.02$.

At posttreatment, PRT was viewed as an acceptable treatment with an average item score of 3.68 on a 5-point scale. ACT was significantly more acceptable, however, $t(65)=3.57, p=.001$, effect size $=0.89$, with an average item score of 4.38 . Because treatment acceptability was necessarily collected at the end of treatment, after there were already outcome differences in the Y-BOCS, this analysis was repeated with pre- to posttreatment Y-BOCS change scores entered as a covariate. ACT continued to 
have significantly higher levels of acceptability, $F(1,64)=5.25$, $p=.025$, effect size $=0.57$. Seventy-five percent of the ACT participants rated the treatment with an average score of 4 or better, and $25 \%$ rated it with the highest acceptability score. Entering treatment credibility, acceptability, and number of treatment sessions as covariates did not change the primary outcomes reported here (results generally became fractionally stronger in favor of ACT).

\section{Discussion}

The purpose of the present study was to examine the applicability, efficiency, and acceptability of a deliberately limited ACT intervention for OCD done without any in-session exposure procedures as compared with PRT. Very limited exclusion criteria were used in this investigation to assess the broad applicability of the approach, and all subtypes of OCD were treated with the same protocol. Both treatments were equally credible, and treatment integrity data were good for both conditions, but results showed greater improvement at posttreatment and at follow-up in OCD symptoms for ACT than for PRT. ACT resulted in a greater number of participants showing clinically significant improvement compared with PRT and had a significantly greater effect on depression among those reporting at least mild depression before treatment. Quality of life improved in both conditions but was marginally in favor of ACT at posttreatment. Finally, psychological constructs thought to be associated with ACT showed greater change in the ACT condition than in PRT, at least initially. All of this suggests that an ACT model appears to be broadly applicable to an OCD population.

\section{Efficiency}

The ACT for OCD protocol that was tested in this investigation is perhaps most notable for its short duration (eight 1-hr sessions) and, thus, for the efficiency of the results produced on a per-unitof-effort basis. The average therapist contact time for ERP and ERP-CT outcome studies (weighted by the size of the study) was $27.4 \mathrm{hr}$ (range: 9-48) for the studies in the review conducted by Abramowitz, Taylor, and McKay (2009). The present study is below the range of durations found in that review (or an earlier review by Fisher \& Wells, 2005) and represents $29 \%-31 \%$ of the average duration of comparable protocols. On average (weighted by the size of the studies), the studies in the Abramowitz, Taylor, and McKay review produced a $45.4 \%$ reduction in the Y-BOCS at posttreatment (follow-up was not reported), which is virtually the same as the $47.3 \%$ reduction in posttreatment and $51.3 \%$ reduction in follow-up seen in the present study. The baseline Y-BOCS scores were very similar as well, with a weighted prescore average of 23.9 in the ERP or ERP-CT groups in the studies in the Abramowitz, Taylor, and McKay review compared with 24.2 in the present study.

We are not arguing that ACT should yet be directly compared with ERP and ERP-CT, nor that research should proceed directly to this question. An ACT approach to OCD is still young; this was a deliberately brief and limited application of an ACT model, and comparisons across study populations need to be done cautiously. Rather, the point is simply that the efficiency suggested by these early results is promising enough that efforts should be made to help ACT for OCD mature. Future studies should examine how processes of change comport with outcomes, how best to structure ACT interventions to move the key processes involved (Should exposure be included? How much intervention is optimal?), and whether ACT can aid ERP and ERP-CT failures. As these questions are answered, the field will be better prepared for the very large and expensive studies that will seemingly be needed to directly compare ACT with existing empirically supported methods on issues of both processes of change and outcomes produced.

\section{Acceptability}

Treatment acceptability was high in both conditions but statistically higher in ACT than in PRT, even after controlling for outcome differences. The acceptability of ACT approached the ceiling of the instrument used (an average of 4.38 out of 5). There were no overt refusals to participate in the ACT condition, either at assignment or during treatment; one ACT participant failed to attend the first session; four more attended fewer than three treatment sessions. Thus five of 41 ACT participants (12.2\%) were categorized as actively or passively declining or dropping out of treatment, which appears to be below the rates expected from recent reviews of ERP and ERP-CT (e.g., Abramowitz, Taylor, \& McKay, 2009).

ACT, as conducted in this trial, did not include in-session exposure exercises. ACT participants were not asked during treatment to approach a feared stimulus, including primary obsessions, and stay in its presence for a period. On the basis of treatment integrity ratings, suggesting that engaging in such activities would be helpful outside of session also never occurred. Participants were taught to learn to see obsessions for what they are, to accept their presence, and to do what is important to them whether or not obsessions were occurring. This is notable, because exposure exercises, though very useful clinically, are met with resistance by a marked number of professionals and clients (Richard \& Gloster, 2007). The absence of these exercises may in part account for the good results seen in treatment acceptability, and thus the present approach may offer an alternative approach to the treatment of OCD when exposure has failed or is declined.

\section{ACT and Exposure}

The absence of in-session exposure in a procedural sense does not mean that exposure in a broader sense was unimportant to the results seen. ACT researchers have long argued that ACT is an exposurebased approach (see Hayes, 1987), and doing exposure in a procedural sense within an ACT approach is generally suggested for anxiety disorders (Eifert \& Forsyth, 2005), even though it was eschewed for strategic reasons in the present study. At the level of process, ACT theory views exposure as organized, values-based contact with previously repertoire-narrowing stimuli for the purpose of producing greater psychological flexibility (Hayes, Strosahl, \& Wilson, in press). In an ACT model, these repertoire-narrowing stimuli are not just external events but also fused thoughts, avoided emotions, experiences that induce inflexible attentional processes, and so on. If that conception is broadly correct, increasing psychological flexibility and values-based action in the presence of difficult emotions and thoughts could well be a key process of change in other successful exposurebased methods. General research will be needed to know whether this is the case. 
At the level of procedure, participants agreed to engage in valuesbased actions that were important and difficult. These generally were important activities that they had stopped engaging in or were only partially engaging in because of attempts to regulate obsessions. Participants were asked to practice the skills learned in the therapy sessions during these activities and to treat them as opportunities to do things that were important to them while more fully acquiring ACT skills. This might be little more than a procedural variant of exposure; if so, it appears to be one with a very high level of acceptability. There are some preliminary data that ACT methods can make exposure more acceptable and less aversive in individuals diagnosed with panic disorder (Levitt et al., 2004), and the same might be true with OCD. Regardless, it is important in future research to understand the processes of change occurring during these exercises and to compare them with those seen in ERP. Thus, the interplay of ACT and exposure at both the process and procedural level should continue to be explored to help better understand both.

\section{Limitations}

There are several limitations to this study. The quality-of-life measure was entirely self-report, and the main outcome measure, though externally rated, was entirely focused on symptom severity. Such a mode of measurement fits uncomfortably with a view that emphasizes function over form and living more effectively over symptom reduction per se. Follow-up was only 3 months due to the preliminary nature of this study, but 6-month to 2-year follow-up is preferable. Assessors were blinded, but penetration of the blind was not formally assessed. Additionally, because the same assessor was used for all participants at all time points to maintain consistency in assessments, the participants were not blind to the timing of assessments. Also, the ACT and PRT conditions could have been matched better if the participants in the PRT condition were asked to use relaxation in response to obsessions, because ACT participants used ACT strategies when obsessions were present. Also, the PRT protocol was briefer than suggested by Bernstein et al. (2000). These weaknesses need to be addressed in future studies. It should also be noted that the sample drawn for this investigation was not ethnically diverse ( $88.6 \%$ Caucasian), thus, extension of the present results in this area should be done with caution. Finally, additional work needs to be conducted to determine the overall effects of the different components and processes within the entire treatment.

The treatment of OCD has progressed substantially since the first treatment trials, but therapists are still far from helping enough individuals in an efficient, acceptable, and broadly effective manner. The development of novel and effective psychosocial interventions such as ACT is needed. It appears that ACT can lead to meaningful clinical outcomes without necessarily using traditional exposure procedures. ACT looks promising as a treatment for OCD, but clearly much more work is needed to develop this approach.

\section{References}

Abramowitz, J. S. (1996). Variants of exposure and response prevention in the treatment of obsessive-compulsive disorder: A meta-analysis. Behavior Therapy, 27, 583-600.

Abramowitz, J. S., Lackey, G. R., \& Wheaton, M. G. (2009). Obsessivecompulsive symptoms: The contribution of obsessional beliefs and experiential avoidance. Journal of Anxiety Disorders, 23, 160-166.
Abramowitz, J. S., Taylor, S., \& McKay, D. (2009). Obsessive-compulsive disorder. Lancet, 374, 491-499.

Arch, J. J., \& Craske, M. G. (2008). Acceptance and commitment therapy and cognitive behavioral therapy for anxiety disorders: Different treatments, similar mechanisms? Clinical Psychology: Science and Practice, $15,263-279$.

Beck, A. T., Steer, R. A., \& Brown, G. K. (1996). Beck Depression Inventory (2nd ed.). San Antonio, TX: Psychological Corporation.

Bernstein, D. A., Borkovec, T. D., \& Hazlett-Stevens, H. (2000). New directions in progressive relaxation training: A guidebook for helping professionals. New York, NY: Praeger.

Bouton, M. E. (2002). Context, ambiguity, and unlearning: Sources of relapse after behavioral extinction. Biological Psychology, 52, 976-986.

Briggs, E. S., \& Price, I. R. (2009). The relationship between adverse childhood experience and obsessive-compulsive symptoms and beliefs: The role of anxiety, depression, and experiential avoidance. Journal of Anxiety Disorders, 23, 1037-1046.

Burckhardt, C. S., Woods, S. L., Schultz, A. A., \& Ziebarth, D. M. (1989). Quality of life of adults with chronic illness: A psychometric study. Research in Nursing \& Health, 12, 347-354.

Cohen, J. (1988). Set correlation and contingency tables. Applied Psychological Measurement, 12, 425-434.

Devilly, G. J., \& Borkovec, T. D. (2000). Psychometric properties of the Credibility/Expectancy Questionnaire. Journal of Behavior Therapy and Experimental Psychiatry, 31, 73-86.

Eifert, G. H., \& Forsyth, J. P. (2005). Acceptance and commitment therapy for anxiety disorders: A practitioner's treatment guide to using mindfulness, acceptance, and values-based behavior change strategies. Oakland, CA: New Harbinger.

Fals-Stewart, W., Marks, A. P., \& Schafer, J. (1993). A comparison of behavioral group therapy and individual behavior therapy in treating obsessive-compulsive disorder. Journal of Nervous and Mental Disease, 181, 189-193.

Feingold, A. (2009). Effect sizes for growth-modeling analysis for controlled clinical trials in the same metric as for classical analysis. Psychological Methods, 14, 43-53.

First, M. B., Spitzer, R. L., Gibbon, M., \& Williams, J. B. W. (2002). Structured Clinical Interview for DSM-IV-TR Axis I disorders, research version, patient edition (SCID-I/P). New York, NY: Biometrics Research, New York State Psychiatric Institute.

Fisher, P. L., \& Wells, A. (2005). How effective are cognitive and behavioral treatments for obsessive-compulsive disorder? A clinical significance analysis. Behaviour Research and Therapy, 43, 1543-1558.

Foa, E. B., Liebowitz, M. R., Kozak, M. J., Davies, S., Campeas, R., Franklin, M. E., . . Tu, X. (2005). Randomized, placebo-controlled trial of exposure and ritual prevention, clomipramine, and their combination in the treatment of obsessive-compulsive disorder. American Journal of Psychiatry, 162, 151-161.

Goodman, W. K., Price, L. H., Rasmussen, S. A., Mazure, C., Fleischmann, R. L., Hill, C. L., . . Charney, D. S. (1989). The Yale-Brown Obsessive Compulsive Scale: I. Development, use, and reliability. Archives of General Psychiatry, 46, 1006-1011.

Hayes, S. C. (1987). A contextual approach to therapeutic change. In N. S. Jacobson (Ed.), Psychotherapists in clinical practice: Cognitive and behavioral perspectives (pp. 327-387). New York, NY: Guilford Press.

Hayes, S. C., Luoma, J. B., Bond, F. W., Masuda, A., \& Lillis, J. (2006). Acceptance and commitment therapy: Model, processes and outcomes. Behaviour Research and Therapy, 44, 1-25.

Hayes, S. C., Strosahl, K. D., \& Wilson, K. G. (1999). Acceptance and commitment therapy: An experiential approach to behavior change. New York, NY: Guilford Press.

Hayes, S. C., Strosahl, K. D., \& Wilson, K. G. (in press). Acceptance and commitment therapy: A unified model of behavior change (2nd ed.). New York, NY: Guilford Press. 
Hayes, S. C., Strosahl, K., Wilson, K. G., Bissett, R. T., Pistorello, J., Toarmino, D.,... McCurry, S. M. (2004). Measuring experiential avoidance: A preliminary test of a working model. Psychological Record, 54, 553-578.

Hedeker, D., \& Gibbons, R. D. (2006). Longitudinal data analysis. Hoboken, $\mathrm{NJ}$ : Wiley-Interscience.

Jacobson, N. S., Follette, W. C., \& Revenstorf, D. (1984). Psychotherapy outcome research: Methods for reporting variability and evaluating clinical significance. Behavior Therapy, 15, 336-352.

Jacobson, N. S., \& Truax, P. (1991). Clinical significance: A statistical approach to defining meaningful change in psychotherapy research. Journal of Consulting and Clinical Psychology, 59, 12-19.

Kelley, M. L., Heffer, R. W., Gresham, F. M., \& Elliott, S. N. (1989). Development of a modified Treatment Evaluation Inventory. Journal of Psychopathology and Behavioral Assessment, 11, 235-247.

Levitt, J. T., Brown, T. A., Orsillo, S. M., \& Barlow, D. H. (2004). The effects of acceptance versus suppression of emotion on subjective and psychophysiological response to carbon dioxide challenge in patients with panic disorder. Behavior Therapy, 35, 747-766.

Manzoni, G. M., Pagnini, F., Castelnuovo, G., \& Molinari, E. (2008). Relaxation training for anxiety: A ten-years systematic review with meta-analysis. BMC Psychiatry, 8(Article 41). doi:10.1186/1471-244X$8-41$

Masuda, A., Hayes, S. C., Sackett, C., \& Twohig, M. P. (2004). Cognitive defusion and self-relevant negative thoughts: Examining the impact of a ninety year old technique. Behaviour Research and Therapy, 42, 477485 .

McLean, P. D., Whittal, M. L., Thordarson, D. S., Taylor, S., Söchting, I., Koch, W. J., ... Anderson, K. W. (2001). Cognitive versus behavior therapy in the group treatment of obsessive-compulsive disorder. Journal of Consulting and Clinical Psychology, 69, 205-214.

Olatunji, B. O., Deacon, B. J., \& Abramowitz, J. S. (2009). The cruelest cure? Ethical issues in the implementation of exposure-based treatments. Cognitive and Behavioral Practice, 16, 172-180.

Rassin, E., Merckelbach, H., Muris, P., \& Schmidt, H. (2001). The Thought-Action Fusion Scale: Further evidence for its reliability and validity. Behaviour Research and Therapy, 39, 537-544.

Reynolds, M., \& Wells, A. (1999). The Thought Control QuestionnairePsychometric properties in a clinical sample, and relationships with PTSD and depression. Psychological Medicine, 29, 1089-1099.

Richard, D. C. S., \& Gloster, A. T. (2007). Exposure therapy has a public relations problem: A dearth of litigation amid a wealth of concern. In D. C. S. Richard \& D. Lauterbach (Eds.), Handbook of the exposure therapies (pp. 409-425). New York, NY: Academic Press.
Segal, D. L., Hersen, M., \& Van Hasselt, V. B. (1994). Reliability of the Structured Clinical Interview for DSM-III-R: An evaluative review. Comprehensive Psychiatry, 35, 316-327.

Shafran, R., Thordarson, D. S., \& Rachman, S. (1996). Thought-action fusion in obsessive compulsive disorder. Journal of Anxiety Disorders, $10,379-391$

Steketee, G., \& Barlow, D. H. (2002). Obsessive-compulsive disorder. In D. H. Barlow (Ed.), Anxiety and its disorders: The nature and treatment of anxiety and panic (2nd ed., pp. 516-550). New York, NY: Guilford Press.

Summerfeldt, L. J., \& Antony, M. M. (2004). Structured and semistructured diagnostic interviews. In M. M. Antony \& D. H. Barlow (Eds.), Handbook of assessment and treatment planning for psychological disorders (pp. 3-37). New York, NY: Guilford Press.

Twohig, M. P., Hayes, S. C., \& Masuda, A. (2006a). Increasing willingness to experience obsessions: Acceptance and commitment therapy as a treatment for obsessive-compulsive disorder. Behavior Therapy, 37, 3-13.

Twohig, M. P., Hayes, S. C., \& Masuda, A. (2006b). A preliminary investigation of acceptance and commitment therapy as a treatment for chronic skin picking. Behaviour Research and Therapy, 44, 1513-1522.

Twohig, M. P., \& Woods, D. W. (2004). A preliminary investigation of acceptance and commitment therapy and habit reversal as a treatment of trichotillomania. Behavior Therapy, 35, 803-820.

Wackerly, D. D., Mendenhall, W., III, \& Scheaffer, R. L. (2008). Mathematical statistics with applications (7th ed.). Pacific Grove, CA: Duxbury Press.

Wells, A., \& Davies, M. I. (1994). The Thought Control Questionnaire: A measure of individual differences in the control of unwanted thoughts. Behaviour Research and Therapy, 32, 871-878.

Wilson, K. G., Sandoz, E. K., Kitchens, J., \& Roberts, M. (2010). The Valued Living Questionnaire: Defining and measuring valued action within a behavioral framework. Psychological Record, 60, 249-272.

Woods, D. W., \& Twohig, M. P. (2008). Trichotillomania: An ACTenhanced behavior therapy approach: Therapist guide. New York, NY: Oxford University Press.

Woods, D. W., Wetterneck, C. T., \& Flessner, C. A. (2006). A controlled evaluation of acceptance and commitment therapy plus habit reversal for trichotillomania. Behaviour Research and Therapy, 44, 639-656.

Received November 17, 2009

Revision received May 27, 2010

Accepted June 11, 2010 